\title{
Risk factors for neck hematoma requiring surgical re-intervention after thyroidectomy: a systematic review and meta-analysis
}

Chunlei Fan ${ }^{1 \dagger}$, Xin Zhou ${ }^{1 \dagger}$, Guoqiang Su${ }^{1 *}$, Yanming Zhou ${ }^{2}$, Jingjun Su${ }^{3^{*}}$, Mingxu Luo ${ }^{1}$ and Hui $\mathrm{Li}^{4}$

\begin{abstract}
Background: In this systematic review and meta-analysis, we aimed to determine the risk factors associated with neck hematoma requiring surgical re-intervention after thyroidectomy.

Methods: We systematically searched all articles available in the literature published in PubMed and CNKI databases through May 30, 2017. The quality of these articles was assessed using the Newcastle-Ottawa Quality Assessment Scale, and data were extracted for classification and analysis by focusing on articles related with neck hematoma requiring surgical re-intervention after thyroidectomy. Our meta-analysis was performed according to the Preferred Reporting Items for Systematic Review and Meta-Analyses guidelines.

Results: Of the 1028 screened articles, 26 met the inclusion criteria and were finally analyzed. The factors associated with a high risk of neck hematoma requiring surgical re-intervention after thyroidectomy included male gender (odds ratio [OR]: 1.86, 95\% confidence interval [Cl]: 1.60-2.17, $P<0.00001$ ), age (MD: 4.92, 95\% Cl: 4.28-5.56, $P<0.00001$ ), Graves disease (OR: 1.81, 95\% Cl: 1.60-2.05, $P<0.00001$ ), hypertension (OR: 2.27, 95\% Cl: 1.43-3.60, $P=0.0005$ ), antithrombotic drug use (OR: 1.92, 95\% Cl: $1.51-2.44, P<0.00001)$, thyroid procedure in low-volume hospitals (OR: 1.32 , $95 \% \mathrm{Cl}: 1.12-1.57, P=0.001)$, prior thyroid surgery (OR: 1.93, 95\% Cl: 1.11-3.37, $P=0.02$ ), bilateral thyroidectomy (OR: $1.19,95 \%$ Cl: 1.09-1.30, $P<0.0001$ ), and neck dissection (OR: 1.55, 95\% Cl: 1.23-1.94, $P=0.0002$ ). Smoking status (OR: 1.19, 95\% Cl: 0.99-1.42, $P=0.06$ ), malignant tumors (OR: 1.00, 95\% Cl: 0.83-1.20, $P=0.97$ ), and drainage used (OR: 2.02, $95 \% \mathrm{Cl}: 0.69-5.89, P=0.20)$ were not significantly associated with postoperative neck hematoma.

Conclusion: We identified certain risk factors for neck hematoma requiring surgical re-intervention after thyroidectomy, including male gender, age, Graves disease, hypertension, antithrombotic agent use, history of thyroid procedures in low-volume hospitals, previous thyroid surgery, bilateral thyroidectomy, and neck dissection. Appropriate intervention measures based on these risk factors may reduce the incidence of postoperative hematoma and yield greater benefits for the patients.
\end{abstract}

Keywords: Risk factor, Hematoma, Bleeding, Thyroidectomy, Thyroid surgery

\footnotetext{
* Correspondence: suquogiang66@163.com; 1208827772@qq.com

${ }^{\dagger}$ Chunlei Fan and Xin Zhou contributed equally to this work.

'Department of Gastrointestinal Surgery III, Xiamen Cancer Hospital, First

Affiliated Hospital of Xiamen University, 55 Zhenhai Road, Xiamen 361003,

China

${ }^{3}$ Department of Ultrasound, First Affiliated Hospital of Xiamen University, 55

Zhenhai Road, Xiamen 361003, China

Full list of author information is available at the end of the article
}

(c) The Author(s). 2019 Open Access This article is distributed under the terms of the Creative Commons Attribution 4.0 International License (http://creativecommons.org/licenses/by/4.0/), which permits unrestricted use, distribution, and reproduction in any medium, provided you give appropriate credit to the original author(s) and the source, provide a link to the Creative Commons license, and indicate if changes were made. The Creative Commons Public Domain Dedication waiver (http://creativecommons.org/publicdomain/zero/1.0/) applies to the data made available in this article, unless otherwise stated. 


\section{Background}

Thyroidectomy is often required for the treatment of malignant thyroid tumors and some benign thyroid diseases [1]. Although thyroid surgery is a relatively safe procedure, it may be associated with some clinically concerning postoperative complications, including postoperative cervical hematoma, incision infection, hypocalcemia, and in some cases vocal cord paralysis. Of these, the development of postoperative cervical hematoma, although rare (incidence: $0.43-6.54 \%$ ) [2-27], may lead to symptoms of compression causing airway obstruction, respiratory distress, or even death due to suffocation.

In recent years, due to the development of new instruments, such as bipolar scalpels, ultrasonic shears, and energy platforms, thyroid surgery has become more precise and prevalent on an outpatient basis as a result of short hospital stays and low costs [28]. However, postoperative cervical hematoma remains a potential lifethreatening complication, and is the main reason why patients are required to stay in the hospital overnight for monitoring after thyroid surgery. To ensure more widespread application of outpatient thyroidectomy and avoid the potential risk of postoperative cervical hematoma, it is vital to determine risk factors that could help clinical surgeons screen patients suitable for outpatient thyroidectomy, and thus minimize the risk of postoperative hematoma [29].

In the present study, we aimed to identify risk factors associated with neck hematoma requiring surgical reintervention after thyroidectomy, including the impact of hypertension, hospital volume, and smoking status.

\section{Methods}

Our meta-analysis was performed according to the Preferred Reporting Items for Systematic Review and MetaAnalyses (PRISMA) guidelines [30].

\section{Study design}

This systematic review and meta-analysis was conducted by reviewing relevant retrospective studies and collecting suitable data.

\section{Search strategies and information sources}

We searched all articles published through May 30, 2017, in the PubMed and CNKI databases by using the following key words: "thyroidectomy and hematoma", "thyroidectomy and hemorrhage", or "thyroidectomy and postoperative bleeding". Two authors conducted the search independently, and any disagreement was resolved by discussion with a third individual.

\section{Study selection}

Studies were included in our meta-analysis using the following criteria: a) retrospective studies; b) articles published in the English or Chinese language; c) studies providing data on the clinical characteristics of the patients; d) studies including patients who underwent open thyroidectomy on either an inpatient or outpatient basis; and e) studies including patients with postoperative hematoma requiring surgical re-intervention.

The exclusion criteria were: a) studies with incomplete data or without data that met our inclusion criteria; b) commentaries, letters, and animal studies; and c) studies including patients receiving minimally invasive thyroidectomy or simple parathyroidectomy.

\section{Data extraction}

After identifying the included articles, 2 reviewers were responsible for extracting the data, including the first author, publication time, design of study, number of patients, and incidence. Patient variables included age, sex, procedure type (unilateral, bilateral, with or without neck dissection), pathology, personal characteristics (Graves disease, hypertension, antithrombotic agent use, smoking status, and previous thyroid surgery), and the use of drainage. A re-extraction was performed by the 2 reviewers together via discussion if debatable data were present in any of the studies.

\section{Quality assessment}

Using the Newcastle Ottawa Scale (NOS) ranging from 0 to 9 [31], all the included studies were assessed for the risk of reporting bias by the 2 reviewers. A study with a score of $\geq 5$ was considered as a study of high quality with a low bias risk; otherwise, it was excluded [32-34]. Any disagreement on the final outcome of assessment was resolved through discussion.

\section{Statistical analysis}

The odds ratio (OR) or mean difference (MD) with a 95\% confidence interval (CI) was chosen as the parameter for this study, and statistical significance was presented as a polled $p$ value $<0.05$. The heterogeneity was measured by the $\mathrm{Q}$ test and $\mathrm{I}^{2}$ statistics. When the heterogeneity test indicated no significant difference $(p>$ 0.1 and $\mathrm{I}^{2}<50 \%$ ), a fixed-effect model was used; otherwise, a random-effects model was used, and the possibility for publication and selective reporting bias was presented using a Begg's funnel plot. All statistical analyses of the data were achieved by using RevMan 5.3 software.

\section{Results}

Study selection

Through a systematic retrieval and manual search of the databases, 1028 articles were screened. The exclusion criteria included duplicated studies, studies with insufficient data, or studies with data that did not meet the 
inclusion criteria. Twenty-six studies were deemed eligible for inclusion in the final analysis. The selection process flow chart for the included studies is shown in Fig. 1.

\section{Study characteristics}

The total number of patients included was 452,799 , and post-thyroidectomy hematoma requiring surgical intervention was noted in 6663 patients. The basic characteristics of the studies included in our meta-analysis are shown in Table 1.

\section{Results of individual analysis \\ Gender}

Twelve studies $[2,3,5-7,10,12,16,19,20,22,23]$ were included for the analysis of hematoma occurrence between males and females. The overall rate of post-thyroidectomy hematoma requiring surgical intervention was $2.10 \%$ in males and $1.27 \%$ in females. Pooled ORs, using a random-effects model $\left(P=0.03, \mathrm{I}^{2}=48 \%\right)$, showed that the occurrence of postoperative hematoma in males was significantly greater than in females (OR: 1.86, 95\% CI: 1.60-2.17, $P<0.00001$; Fig. 2a).
Age

We used a fixed-effects model to analyze the impact of age on the occurrence of postoperative hematoma $(P=$ $0.74, \mathrm{I}^{2}=0 \%$ ). A total of 5 studies $[3,4,7,10,12,22]$ were included, which exhibited a significant difference between the hematoma and non-hematoma groups (MD: 4.92, 95\% CI: 4.28-5.56, $P<0.00001$; Fig. 2b). The $\mathrm{I}^{2}$ demonstrated that there was no significant heterogeneity between the studies included in the analysis.

\section{Graves disease}

A fixed-effects model was used for data analysis $(P=$ $0.21, \mathrm{I}^{2}=28 \%$ ), and 8 studies $[2,9,12,15,16,22,26,27]$ were included in the analysis. There was a significantly higher incidence of post-thyroidectomy hematoma in patients with Graves disease (OR: 1.81, 95\%CI: 1.60 2.05, $P<0.00001$; Fig. 2c).

\section{Hypertension}

We used a random-effects model for analysis due to the heterogeneity of the data $\left(P<0.00001, \mathrm{I}^{2}=92 \%\right)$. Four studies $[2,6,10,19]$ were included in the analysis. The incidence of postoperative hematoma was significantly

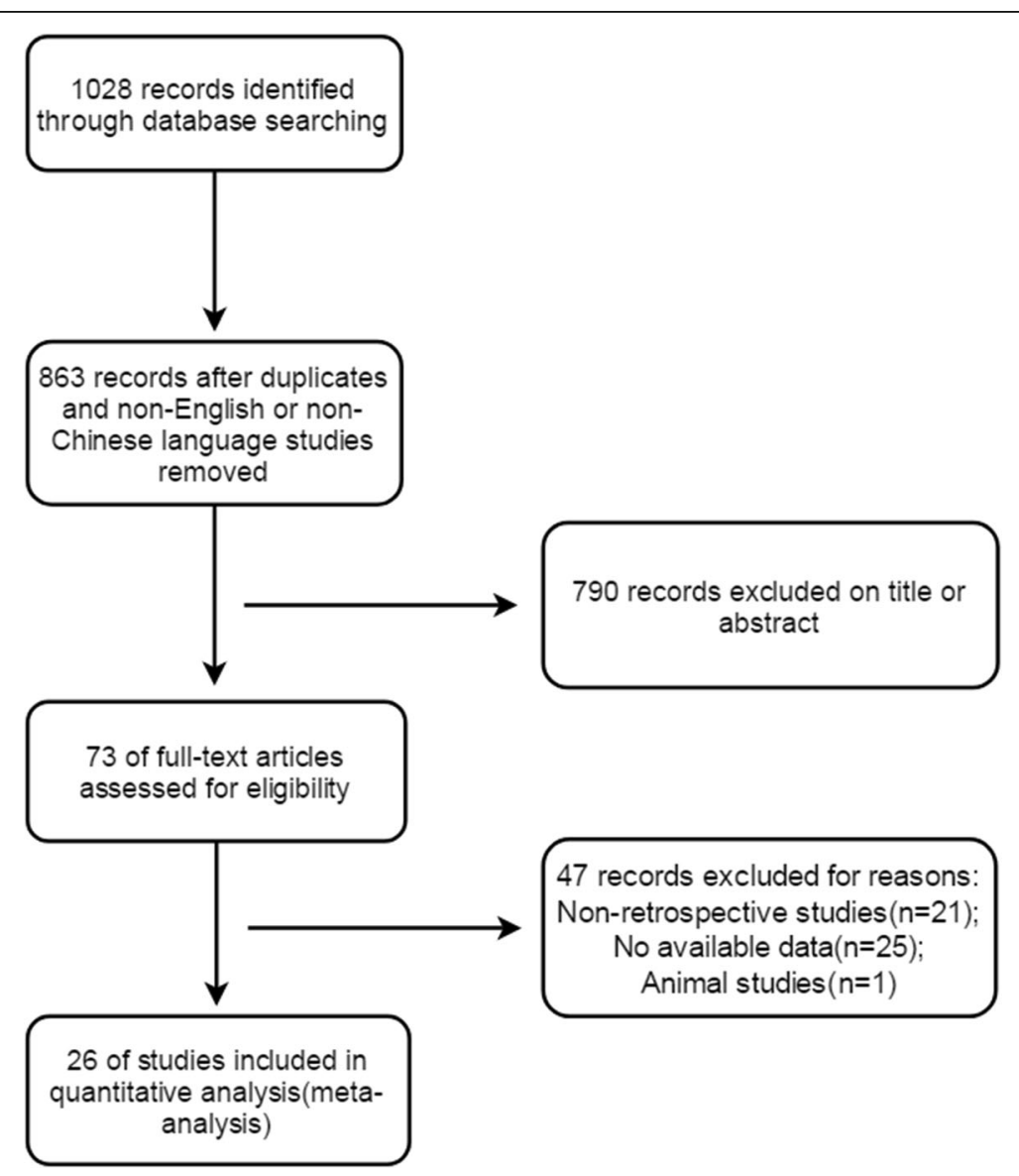

Fig. 1 Study flow diagram of the meta-analysis 
Table 1 The basic characteristics of the included studies

\begin{tabular}{|c|c|c|c|c|c|c|c|c|c|c|c|c|}
\hline Author & Year & $\begin{array}{l}\text { Type of } \\
\text { study }\end{array}$ & $\begin{array}{l}\text { Country/ } \\
\text { Region }\end{array}$ & $\begin{array}{l}\text { NOS } \\
\text { score }\end{array}$ & Age(Mean) & $\operatorname{Sex}(M / F)$ & No.Graves & No.Cancer & No.Others & Procedure & $\begin{array}{l}\text { Hematoma/ } \\
\text { Total }\end{array}$ & Rate(\%) \\
\hline Liu,J. & 2016 & Retrospective & China & 7 & NA & $\begin{array}{l}1401 / \\
3755\end{array}$ & 0 & 4167 & 989 & $\pi \mathrm{T}, \mathrm{HT}$ & $44 / 5156$ & 0.85 \\
\hline Suzuki,S. & 2016 & Retrospective & Japan & 7 & NA & $\begin{array}{l}1967 / \\
40000\end{array}$ & 3383 & 29715 & 18869 & $\Pi \mathrm{T}, \mathrm{HT}$ & $920 / 51967$ & 1.77 \\
\hline $\begin{array}{l}\text { Narayanan, } \\
\text { S. }\end{array}$ & 2016 & Retrospective & USA & 7 & 51.2 & $271 / 1176$ & NA & 441 & NA & $\Pi \mathrm{T}, \mathrm{HT}$ & 10/1447 & 0.69 \\
\hline $\begin{array}{l}\text { Oltmann, } \\
\text { S.C. }\end{array}$ & 2016 & Retrospective & USA & 7 & 49 & $527 / 1868$ & NA & NA & NA & $\Pi \mathrm{T}, \mathrm{HT}$ & 20/2395 & 0.84 \\
\hline Perera,M. & 2016 & Retrospective & Australia & 7 & 51.6 & $41 / 164$ & NA & 38 & NA & $\Pi \mathrm{T}, \mathrm{HT}$ & $9 / 205$ & 4.39 \\
\hline $\begin{array}{l}\text { Sorensen, } \\
\text { K.R. }\end{array}$ & 2015 & Retrospective & Denmark & 7 & 53.2 & $378 / 1134$ & NA & 309 & NA & $\mathrm{TT}, \mathrm{HT}$ & $42 / 1512$ & 2.77 \\
\hline Dehal,A. & 2015 & Retrospective & USA & 7 & NA & $\begin{array}{l}32033 / \\
145124\end{array}$ & 4342 & 37038 & 105954 & $\Pi \mathrm{T}, \mathrm{HT}$ & $\begin{array}{l}2210 / \\
147334\end{array}$ & 1.49 \\
\hline $\begin{array}{l}\text { Hardman, } \\
\text { J.C. }\end{array}$ & 2015 & Retrospective & UK & 6 & 46.3 & $341 / 1316$ & NA & NA & NA & $\Pi \mathrm{T}, \mathrm{HT}$ & $32 / 1657$ & 1.93 \\
\hline Chen,E. & 2014 & Retrospective & China & 7 & 46.1 & $872 / 3577$ & 296 & 2027 & 2176 & $\Pi \mathrm{T}, \mathrm{HT}$ & $88 / 4499$ & 1.96 \\
\hline Dixon,J.L. & 2014 & Retrospective & USA & 7 & NA & NA & NA & NA & NA & $\Pi \mathrm{T}, \mathrm{HT}$ & $18 / 4140$ & 0.43 \\
\hline Weiss,A. & 2014 & Retrospective & USA & 7 & 52 & $\begin{array}{l}31186 / \\
118826\end{array}$ & NA & 46298 & NA & $\mathrm{TT}, \mathrm{HT}$ & $\begin{array}{l}1870 / \\
150012\end{array}$ & 1.25 \\
\hline Vassiliou,l. & 2013 & Retrospective & Greece & 7 & 54 & $63 / 153$ & 2 & 100 & 214 & $\pi$ & $3 / 216$ & 1.39 \\
\hline Kandil,E. & 2013 & Retrospective & USA & 7 & NA & NA & 1197 & 9199 & 11229 & $\pi$ & $366 / 21625$ & 1.69 \\
\hline Calo,P.G. & 2012 & Retrospective & Italy & 7 & NA & $8 / 98$ & NA & 10 & NA & $\Pi \mathrm{T}, \mathrm{HT}$ & $3 / 106$ & 2.83 \\
\hline $\begin{array}{l}\text { Promberger, } \\
\text { R. }\end{array}$ & 2012 & Retrospective & Austria & 7 & NA & $\begin{array}{l}5727 / \\
24415\end{array}$ & 1052 & 2460 & 36630 & $\mathrm{TT}, \mathrm{HT}$ & $519 / 30142$ & 1.72 \\
\hline Agarwal,A. & 2012 & Retrospective & India & 7 & 42.7 & $244 / 569$ & NA & 266 & NA & $\pi$ & $6 / 813$ & 0.74 \\
\hline Lang,B.H. & 2012 & Retrospective & Hongkong & 7 & 48 & $569 / 2517$ & 415 & 554 & 2117 & $\mathrm{TT}, \mathrm{HT}$ & $22 / 3086$ & 0.71 \\
\hline Calo,P,G. & 2010 & Retrospective & Italy & 7 & 56 & $465 / 2094$ & 259 & 799 & 1501 & $\Pi \mathrm{T}, \mathrm{HT}$ & $32 / 2559$ & 1.25 \\
\hline Godballe,C. & 2009 & Retrospective & Denmark & 7 & 50 & $\begin{array}{l}1147 / \\
4343\end{array}$ & NA & 774 & NA & TT,HT & $230 / 5422$ & 4.24 \\
\hline Shih,M.L. & 2008 & Retrospective & USA & 7 & 45.4 & $82 / 392$ & NA & 133 & NA & $\mathrm{TT}, \mathrm{HT}$ & $4 / 474$ & 0.84 \\
\hline Leyre,P. & 2008 & Retrospective & France & 7 & 54 & $\begin{array}{l}1112 / \\
5718\end{array}$ & 406 & NA & NA & $\Pi \mathrm{T}, \mathrm{HT}$ & $70 / 6830$ & 1.02 \\
\hline $\begin{array}{l}\text { Bergenfelz, } \\
\text { A. }\end{array}$ & 2008 & Retrospective & Sweden & 7 & NA & $659 / 3001$ & 659 & 450 & 2551 & $\Pi \mathrm{T}, \mathrm{HT}$ & $76 / 3660$ & 2.07 \\
\hline Lefevre,J.H. & 2007 & Retrospective & France & 7 & 51 & $108 / 577$ & NA & 92 & NA & $\mathrm{TT}, \mathrm{HT}$ & $6 / 685$ & 0.88 \\
\hline Chiang,F.Y. & 2006 & Retrospective & Taiwan & 6 & NA & NA & 48 & NA & NA & $\pi$ & $7 / 107$ & 6.54 \\
\hline Ozlem,N. & 2006 & Retrospective & Turkey & 7 & 38 & $180 / 886$ & NA & NA & NA & $\Pi \mathrm{T}, \mathrm{HT}$ & $5 / 1066$ & 0.47 \\
\hline Gaujoux,S. & 2006 & Retrospective & France & 7 & 49 & $894 / 4246$ & 714 & NA & NA & $\Pi \mathrm{T}, \mathrm{HT}$ & $51 / 5140$ & 0.99 \\
\hline
\end{tabular}

NOS Newcastle Ottawa Scale, No. Number, $M$ Male, $F$ Female, NA Data not available, $\Pi T$ Total thyroidectomy; $H T$ Hemi-/subtotal/partial thyroidectomy

greater in the patients with hypertension (OR: $2.27,95 \%$ CI: $1.44-3.68, P=0.0005$; Fig. $2 \mathrm{~d}$ ).

\section{Antithrombotic drug use}

Data concerning the use of antithrombotic drugs were analyzed using a fixed-effects model $\left(P=0.20, \mathrm{I}^{2}=32 \%\right)$. All patients undergoing antithrombotic therapy before the operation were included in this meta-analysis, regardless of the medication route and dosage. The results showed that the occurrence of post-thyroidectomy hematoma in patients using antithrombotic drugs was significantly higher than that in patients who did not use antithrombotic drugs (OR: 1.92, 95\% CI: 1.512.44, $P<0.00001$; Fig. 3a).

\section{Hospital volume}

Hospital volumes in terms of thyroidectomies performed were classified as low ( $<75$ per year) and high $(\geq 75$ per year). Data analysis using the random-effects model $\left(P=0.0001, \mathrm{I}^{2}=83 \%\right)$ showed that thyroidectomies 


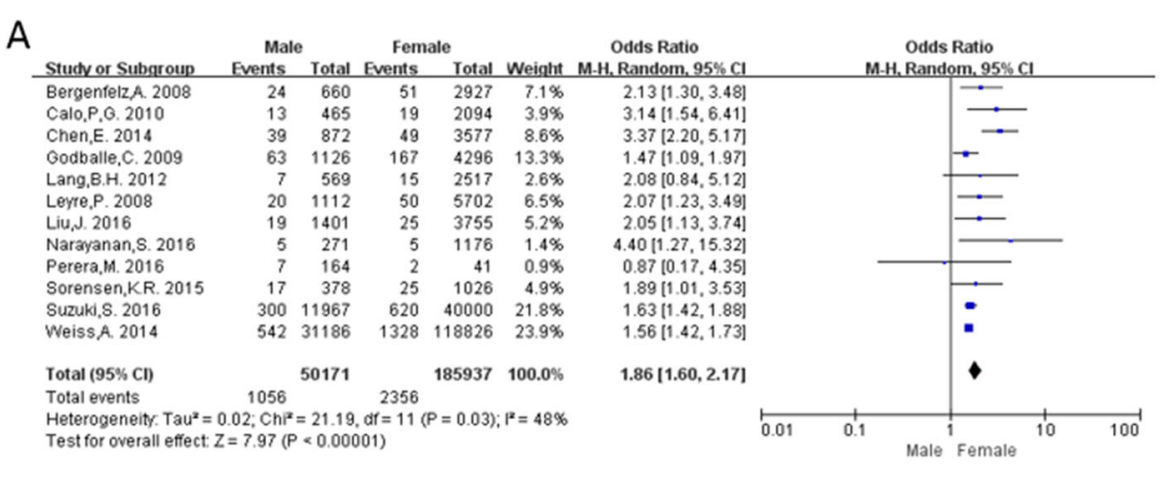

B

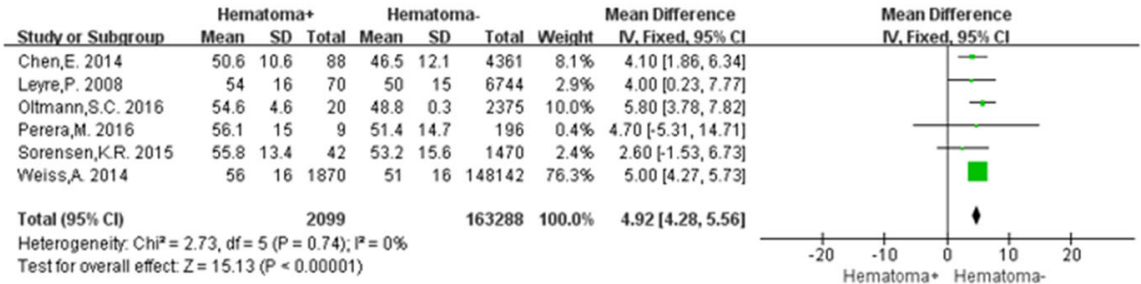

C

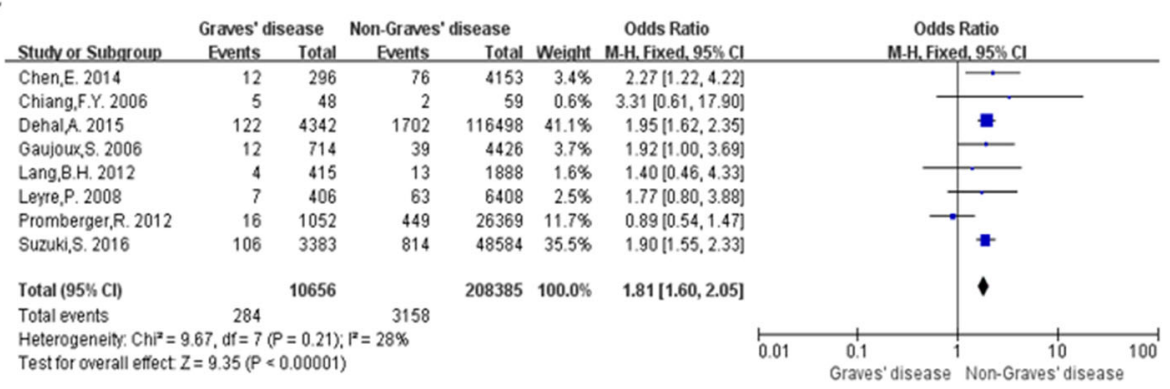

D

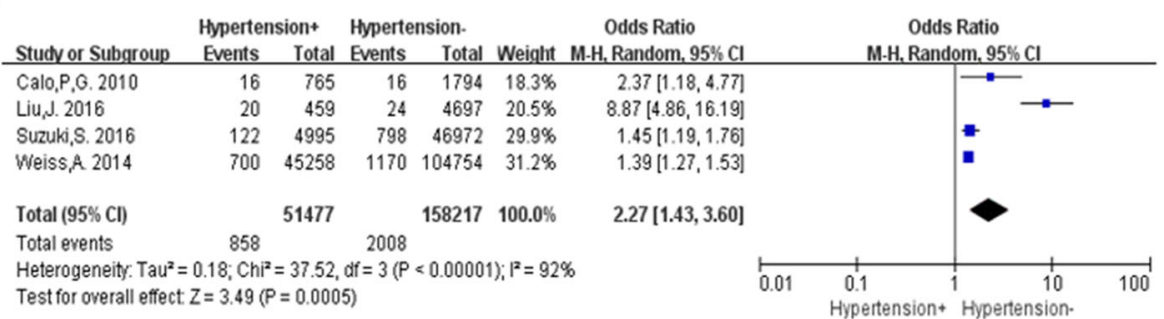

Fig. 2 Meta-analysis results of post-thyroidectomy hematoma between the 2 groups. a Gender; b Age; c Graves disease; d Hypertension

performed in low-volume hospitals had a higher propensity to result in postoperative hematoma, as compared to those performed in high-volume hospitals (OR: 1.32, 95\% CI: 1.12-1.57, $P=0.001$; Fig. 3b).

\section{Previous thyroid operation}

A random-effects model was used to conduct the data analysis $\left(P=0.003, \mathrm{I}^{2}=69 \%\right)$. Seven studies $[6-8,16,17,20,24]$ were included in this analysis. The occurrence of post-thyroidectomy hematoma was higher in patients with a history of thyroidectomy than in patients without (OR: 1.93, 95\% CI: 1.113.37, $P=0.02$; Fig. $3 \mathrm{c}$ ).

\section{Surgical extent}

Based on the extent of surgery, patients were divided into 2 groups: the bilateral surgery group and unilateral surgery group. Data analysis using a fixed-effects model $\left(P=0.12, \mathrm{I}^{2}=40 \%\right)$ showed that postoperative hematoma was more likely to occur in patients receiving bilateral thyroidectomy than in patients receiving unilateral surgery (OR: 1.19, 95\% CI: $1.09-$ 1.30, $P<0.0001$; Fig. 3d). 
A

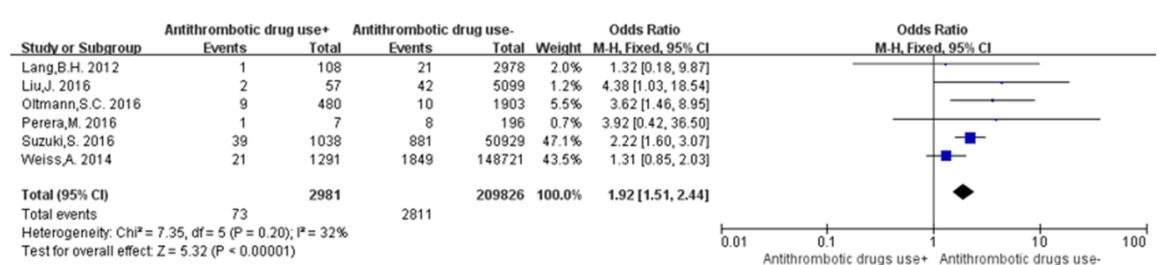

B

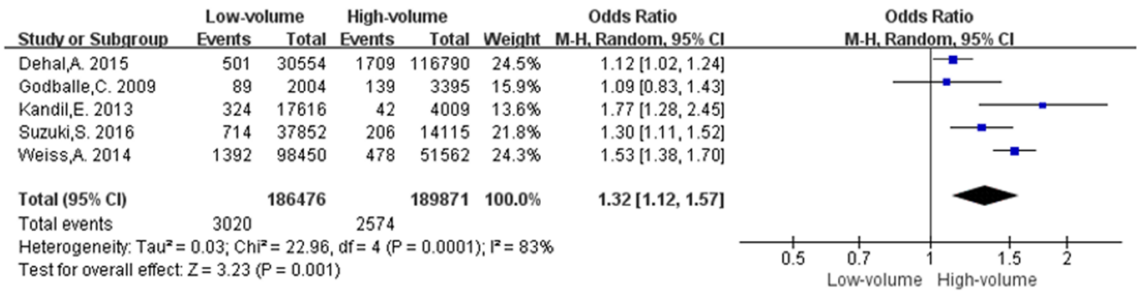

C
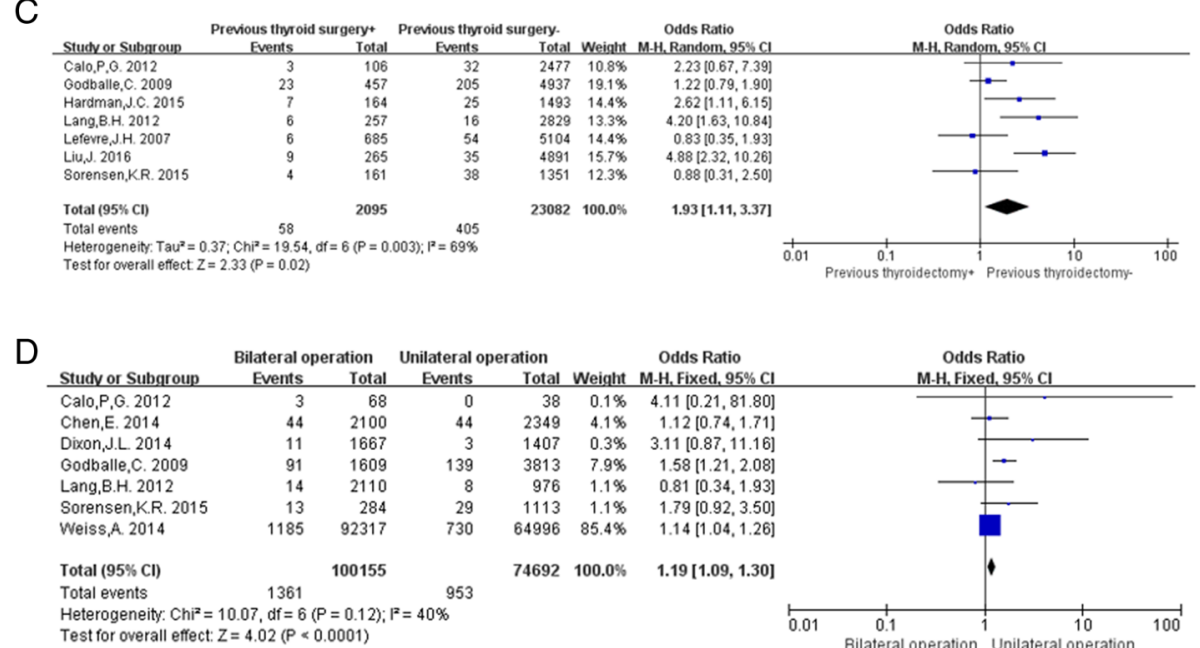

Fig. 3 Meta-analysis results of post-thyroidectomy hematoma between the 2 groups. a Antithrombotic drug use; b Hospital volume; c Previous thyroid surgery; d Extent of operation

\section{Neck dissection}

Data analysis of neck dissection using a random-effects model $\left(P=0.09, \mathrm{I}^{2}=48 \%\right)$ showed that postoperative hematoma was more likely to occur in patients who underwent neck dissection than in those who did not (OR: 1.55, 95\% CI: 1.23-1.94, $P=0.0002$; Fig. 4a).

\section{Smoking status}

A random-effects model was used for this analysis because of the high heterogeneity in the collected data $\left(P=0.02, \mathrm{I}^{2}=71 \%\right)$. The results showed that there was no significant increase in the incidence of post-thyroidectomy hematoma in patients who smoked (OR: 1.19, 95\% CI: 0.99-1.42, $P=0.06$; Fig. 4b).

\section{Tumor characteristics}

Twelve studies [6-10,12,13,15, 18-21] were included for this analysis by using a random-effects model $t$ test
$\left(P=0.0007, \mathrm{I}^{2}=66 \%\right)$. The tumor was classified as malignant or benign, and we found that the tumor characteristics were not associated with the risk of postoperative hematoma (OR: 1.00, 95\% CI: 0.83-1.20, $P=0.97$; Fig. 4c).

\section{Drain placement}

Data concerning drain placement were extracted from 6 studies and a random-effects model was used to analyze the data $\left(P<0.00001, \mathrm{I}^{2}=86 \%\right)$. The results showed that the placement of drain devices was not associated with the occurrence of post-thyroidectomy hematoma (OR: 2.02, 95\% CI: 0.69-5.89, $P=0.20$; Fig. 4d).

Pooled outcomes of all factors are shown in Table 2.

\section{Discussion}

Postoperative hematoma is a challenging complication after thyroidectomy, particularly during the period following out-patient surgery. Cervical hematoma formation 

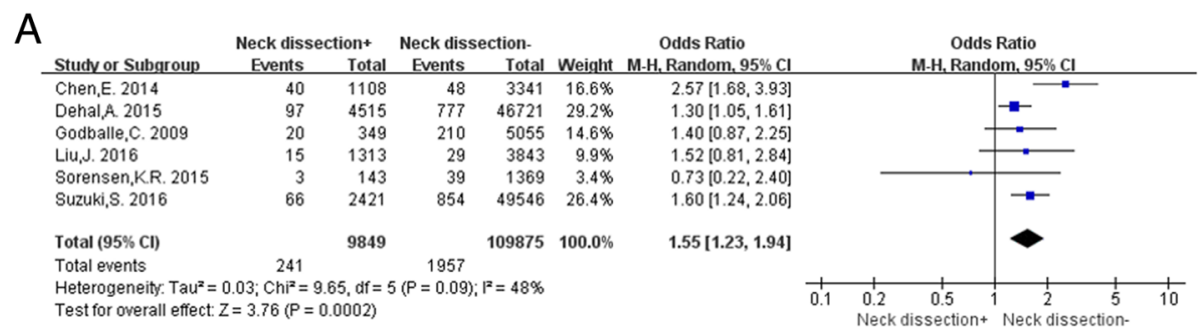

B

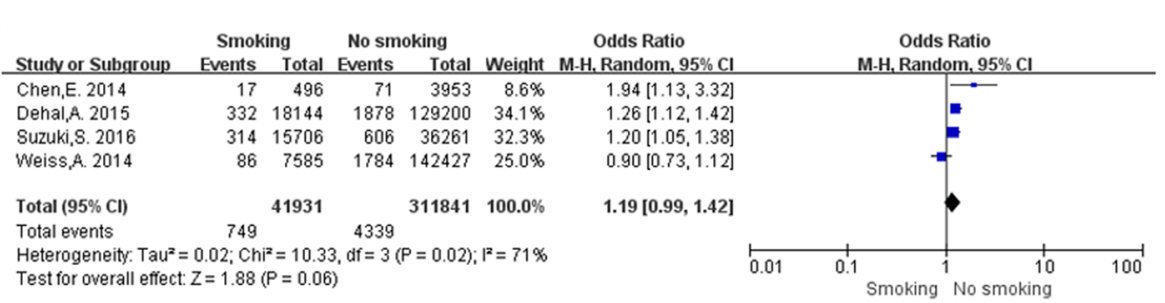

C
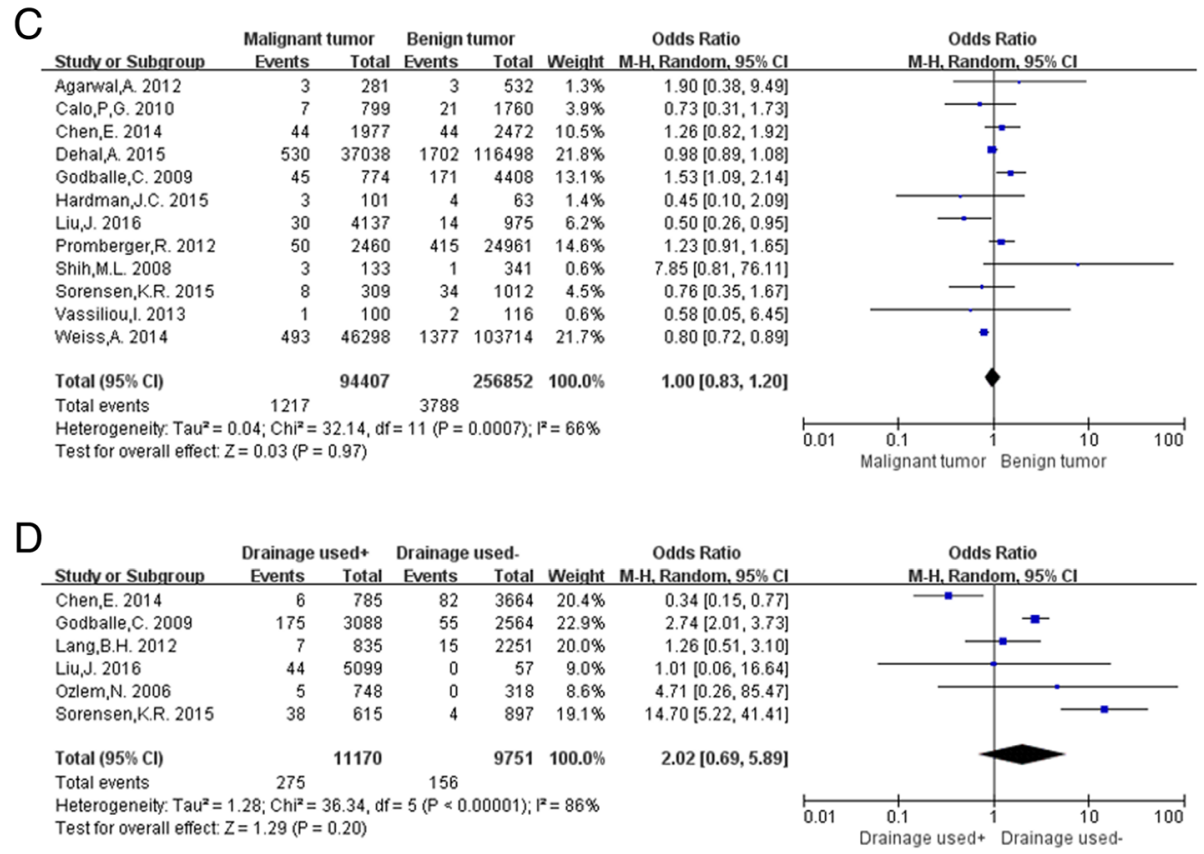

Fig. 4 Meta-analysis results of post-thyroidectomy hematoma between the 2 groups. a Neck dissection; b Smoking status; c Tumor character; d Drainage used

may lead to airway obstruction or respiratory distress in some cases, and immediate surgical re-intervention is required to avoid asphyxia, cardiac arrest, or even death. Although post-thyroidectomy hematoma is a rare complication, it may be potentially life-threatening once the symptoms develop. As a large proportion of thyroid surgery procedures are performed on an outpatient basis, the avoidance of postoperative hematoma has become a major concern for clinical surgeons [28]. Therefore, we pooled the results of previous studies to identify potential risk factors associated with neck hematoma requiring surgical intervention after thyroidectomy.
Our meta-analysis included 50,171 males and 185,937 females undergoing thyroidectomy for various types of thyroid diseases. The risk of post-thyroidectomy hematoma was 1.86-fold higher in males than in females (OR: 1.86, 95\% CI: 1.60-2.17, $P<0.00001)$. The reason for the different incidence between males and females is unclear, but we infer that there are 3 possible causes. First, blood vessels in males are thicker than in females, and therefore, blood flow in the thyroid glands is more abundant. When hemostasis in the neck area is inadequate or surgical ligation loosens, thick vessels can generate more vascular hemorrhage than thin vessels, which increases the likelihood of neck 
Table 2 Pooled outcomes of all factors

\begin{tabular}{|c|c|c|c|c|c|c|}
\hline Factors & No. of studies & Statistical model & $\mathrm{MD} / \mathrm{OR}$ & $95 \% \mathrm{Cl}$ & $P$ vaule & Heterogeneity \\
\hline Male & 12 & random-effects & 1.86 & $1.60-2.17$ & $<0.00001$ & $P=0.03, I^{2}=48 \%$ \\
\hline Age & 6 & fixed-effects & 4.92 & $4.28-5.56$ & $<0.00001$ & $P=0.74,1^{2}=0 \%$ \\
\hline Graves Disease & 8 & fixed-effects & 1.81 & $1.60-2.05$ & $<0.00001$ & $P=0.21,\left.\right|^{2}=28 \%$ \\
\hline Hypertension & 4 & random-effects & 2.27 & $1.43-3.60$ & 0.0005 & $P<0.00001, I^{2}=92 \%$ \\
\hline Antithrombotic Drug used & 6 & fixed-effects & 1.92 & $1.51-2.44$ & $<0.00001$ & $P=0.20, I^{2}=32 \%$ \\
\hline Low-volume hospital & 5 & random-effects & 1.32 & $1.12-1.57$ & 0.001 & $P=0.0001, I^{2}=83 \%$ \\
\hline Previous thyroid surgey & 7 & random-effects & 1.93 & $1.11-3.37$ & 0.02 & $P=0.003, I^{2}=69 \%$ \\
\hline Bilateral thyroidectomy & 7 & fixed-effects & 1.19 & $1.09-1.30$ & $<0.0001$ & $P=0.12, I^{2}=40 \%$ \\
\hline Neck dissection & 6 & random-effects & 1.55 & $1.23-1.94$ & 0.0002 & $P=0.09, I^{2}=48 \%$ \\
\hline Smoking status & 4 & random-effects & 1.19 & $0.99-1.42$ & 0.06 & $P=0.02, I^{2}=71 \%$ \\
\hline Malignant tumor & 12 & random-effects & 1.00 & $0.83-1.20$ & 0.97 & $P=0.0007, I^{2}=66 \%$ \\
\hline Drainage used & 6 & random-effects & 2.02 & $0.69-5.89$ & 0.20 & $P<0.00001, I^{2}=86 \%$ \\
\hline
\end{tabular}

hematoma in males. In addition, smoking, drinking, and hypertension are more common in males [19, 35], all of which can cause vascular changes such as increased vascular brittleness or decreased coagulation. When neck stimulation, such as in emesis, cough, or abrupt neck activity, occurs, vascular rupture and bleeding are more likely to occur, which leads to the formation of hematoma. Finally, some studies suggest that male gender is one of the risk factors of thyroid cancer and males are hence more prone to develop lymph node metastasis [36, 37]; therefore, thyroid surgery may be more complex and the extent of surgery may be wider, and blood vessel damage and bleeding could be greater. This could be a contributing factor for the formation of hematoma.

Several previous articles have reported that age is a risk factor for post-thyroidectomy neck hematoma $[3,4,7,10,12,22]$. In the present study, the average patient age ranged from 46.5 to 56.1 years; the mean age of the hematoma group was greater than that of the non-hematoma group (MD: 4.92, 95\%CI: 4.28$5.56, P<0.00001)$, which suggests that older patients in this age range had a higher risk of suffering postoperative hematoma. This conclusion is consistent with the previous results. We believe that this outcome can be attributed to the increased vascular brittleness in older patients. As individuals age, the blood vessels may become less elastic, which may likely contribute to angiorhagia and decreased vasoconstriction. Eventually, neck hemorrhage or hematoma may occur. However, as the age divisions in previous studies are not consistent, we could not determine which age bracket was most prone to postoperative neck hematoma.

At present, the indications of thyroid surgery for Graves disease include compressive symptoms, uncontrollable hyperthyroidism, large goiter, coexisting malignant tumors, retrosternal or substernal extension, females planning pregnancy, and Graves ophthalmopathy [38]. The advantage of surgery for Graves disease is that definitive treatment can be provided, and relative symptoms can be rapidly alleviated. However, Graves disease is an autoimmune thyroid condition, and the thyroid glands of patients with Graves disease are rich in vascularity [39]. This type of physiological change may increase the risk of intraoperative hemorrhage and may aggravate the influence of surgical vision, which could enhance the difficulty of the surgery or may even lead to an increased incidence of complications. Previous research has shown that using inorganic iodine for several days prior to surgery can decrease intraoperative blood loss by reducing thyroid hormone release and thyroid vascularity, and should hence be recommended for most patients undergoing thyroidectomy for Graves disease [40]. However, most studies included in our research did not include a detailed description of the use of inorganic iodine before surgery, and did not explain whether this preoperative therapy, if regularly performed, could affect the risk of postoperative hematoma in Graves disease. In the present study, treatment with inorganic iodine had to be ignored due to the lack of detailed information. Therefore, we found that there was an increased rate of hematoma formation following thyroidectomy for Graves disease, as compared to other indications for thyroidectomy (OR: 1.81, 95\% CI: 1.60-2.05, $P<0.00001$ ).

Some researchers believe that postoperative hematoma formation in most cases was probably due to postoperative hypertension (SP $>150 \mathrm{mmHg}$ ) [41]. Hypertension may cause vascular stiffness, which increases the likelihood of postoperative hemorrhage; in particular, elevated blood pressure after surgery could increase the risk of hematoma formation. Hence, close monitoring of blood pressure during the first $24 \mathrm{~h}$ after surgery and prompt treatment of all manifestations of hypertension with 
appropriate drugs are recommended [42]. In the present study, we observed an increased risk of hematoma in patients with hypertension (OR: 2.27, 95\% CI: $1.43-3.60, P=0.0005)$. Therefore, care should be taken during the postoperative period in patients with hypertension, particularly in patients in whom antihypertensive therapy has been discontinued preoperatively due to anesthesia. Smooth extubation without significant retching or coughing, as well as the control of both postoperative vomiting and pain to avoid an increase in venous or arterial pressure, are important considerations for minimizing the risk of post-thyroidectomy hematoma [35, 43, 44].

The preoperative use of antiplatelet and/or anticoagulant medications may be another potential risk factor for post-thyroidectomy hematoma. The indications and use of these medications are expanding in older populations [45], as these drugs have been proven to prevent clot formation or platelet aggregation, both of which can affect postoperative hemostasis. Therefore, some surgeons maintain that avoiding antithrombotic drug use 1 week before surgery may decrease the risk of postoperative hemorrhage; these antithrombotic drugs include anticoagulant agents (warfarin, edoxaban, apixaban, rivaroxaban, and dabigatran) and antiplatelet agents (aspirin, clopidogrel, ticlopidine, and cilostazol) [46]. Nevertheless, it is unclear whether the use of these antithrombotic drugs should be suspended and whether a 1-week period is sufficient. In the present analysis, we included all patients who used antithrombotic drugs, regardless of the drug type, dosage, route of medication, and drug outage time. The results of statistical analysis indicated that patients receiving antithrombotic medications had an increased risk of hematoma formation, compared with those not receiving these medications (OR: 1.92 , 95\% CI: 1.51-2.44, $P<0.00001)$. Due to our limited dataset, we were unable to perform subgroup analysis about the drug type, dosage, route of medication, and drug outage time. Hence, further studies are needed to clarify the impact of these drugs on the risk of postoperative hematoma formation.

Moreover, our research analyzed the relationship between the volume of hospitals and the occurrence of postoperative hematoma. We divided the hospitals into high-volume and low-volume hospitals. High-volume hospitals were defined as centers that performed $\geq 75$ thyroidectomies per year on average, and low-volume hospitals were defined as centers that performed as $<75$ thyroidectomies per year. We found that more cases of post-thyroidectomy hematoma occurred in low-volume hospitals (OR: 1.32, 95\% CI: $1.12-1.57, P=0.001$ ). The most common explanation for this relationship could be that both surgeon and hospital experience is related to improved patient care-i.e., "practice makes perfect". Some investigators have noted an independent effect of hospital volume [47], whereas other researchers found that most of the volume-outcome relation can be explained by surgeon experience [48, 49]. Surgeons in high-volume hospitals may have more experience with thyroid surgery and may be better at blood vessel ligature or intraoperative hemostasis, which may explain the low incidence of hematoma in high-volume hospitals.

Re-operative thyroid surgery can be challenging for surgeons. During the primary thyroid surgery, removal of part or the entire gland could distort the anatomy and cause postoperative tissue changes through scarring and fibrosis [50]. Radioactive iodine treatment can make tissues stiffer, woodier, and more likely to bleed [34]. The second or third thyroidectomy in these cases will involve dissection through previously disturbed tissues, which could contribute to a higher hematoma rate. Our study showed that previous thyroid surgery was a risk factor for post-thyroidectomy hematoma (OR: 1.93, 95\% CI: $1.11-3.37, P=0.02)[6-8,16,17,20,24]$. Thyroidectomy in patients who have undergone a previous thyroid operation may therefore be safely considered by experienced surgeons.

Hematoma is more likely to occur in patients undergoing a more extensive surgical resection. Compared with unilateral thyroidectomy, bilateral thyroidectomy results in a larger wound and greater tissue injury. Larger wounds and more severe tissue injury may greatly increase the possibility of postoperative hematoma (OR: 1.19, 95\% CI: $1.09-1.30, P<0.0001)$. This explanation may also be responsible for the higher incidence of postoperative hematoma in patients with neck dissection (OR: 1.55, 95\% CI: 1.23-1.94, $P=0.0002$ ). Compared with simple thyroidectomy, neck dissection involves an additional procedure that requires resection over a larger anatomical area and may be associated with larger injury to the cervical muscles and surrounding blood vessels. A large dead space is often formed following neck dissection and facilitates hematoma formation [12]. Cervical muscles are a common postoperative bleeding site, and hence, they need to be stretched to obtain an essential amount of operating space and surgical vision during thyroidectomy, particularly when deep lymph node dissection is performed. This may lead to injury to small blood vessels on the surface of the muscle, and may increase the risk of postoperative hematoma formation. To avoid overlooking imperceptible bleeding points, a meticulous surgical technique with careful hemostasis is necessary.

It is unclear whether smoking status is a risk factor for post-thyroidectomy hematoma. Some researchers suggested that smokers had an increased postoperative bleeding tendency $[19,35]$, but others argued that smoking status was not an independent risk factor. Weiss et al. $[2,9,10,12]$ found no association between smoking 
status and postoperative hematoma, consistent with our conclusion (OR: 1.19, 95\% CI: 0.99-1.42, $P=$ 0.06). In the smokers in the present study, none of the included studies provided a relative description of the number of cigarettes smoked per day. We ignored the amount of cigarettes smoked, and defined smoking status as smoker (current or former smoker) and never smoker.

Our univariate analysis showed no significant correlation between the smoking status and postoperative hematoma, although the risk of neck hematoma was increased in patients with chronic obstructive pulmonary disease (COPD), diabetes, chronic renal disease, and other underlying diseases with a comorbidity score of $\geq 3$ [9]. Due to the limited data, we were unable to further analyze the comorbidities; however, it is important to control the comorbidities before the operation.

To analyze the relationship between the nature of thyroid tumors and postoperative hematoma, we divided the tumors into benign and malignant, and found that there was no significant difference between them in terms of postoperative hematoma formation (OR: $1.00,95 \%$ CI: $0.83-1.20, P=0.97$ ), inconsistent with previous studies $[2,6]$. In several included studies, some researchers classified benign thyroid lesions as benign tumors, which could have affected the outcome. Moreover, in some patients, the final pathological diagnosis may differ from the intraoperative frozen section diagnosis, which could have affected the result.

Drain device placement after thyroidectomy is a routine procedure to drain possible postoperative hemorrhage, which could lead to cervical hematoma formation or even airway compression [51]. Our analysis indicated that routine placement of drain devices did not significantly increase the risk of postoperative hematoma (OR: 2.02, 95\% CI: $0.69-5.89, P=0.20$ ). However, previous studies showed that this prevention measure did not have significant postoperative advantages because it could have increased the incidence of infective complications and led to a longer hospital stay [52-54]. Based on these findings, we concluded that although routine drain device placement is a not a risk factor for postoperative hematoma formation, meticulous intra-operative hemostasis should never be ignored.

Besides the above-mentioned analysis, diabetes, alcohol abuse, high body mass index, obesity, multiple nodules, thyroid weight, substernal goiter, history of iodine therapy, anesthesia methods, and new types of hemostatic equipment may be related to postoperative hematoma. However, due to the insufficiency of the literature, we were unable to include all these factors into our meta-analysis. To clarify these factors, additional relative research will be required.

\section{Limitations}

Our meta-analysis has certain limitations. First, in some studies, the definition of hematoma was not clear. Therefore, we clearly defined hematoma as patients who require surgical re-intervention, including return to the operating room or need for bedside monitoring with incision of the skin and evacuation of hematoma. Consequently, studies that did not meet this inclusion criteria were excluded, which means that our analysis may have underestimated the overall incidence of hematoma. Second, only 4 articles meeting the inclusion criteria analyzed the correlation of hypertension and smoking status with postoperative hematoma formation, and hence, the resulting conclusion may not be sufficiently convincing. Third, in the 4 articles that analyzed the volume of hospitals, the definition of hospital volume was inconsistent, and we had to classify hospitals as high-volume and low-volume, which could affect the inconsistency between the conclusion and the objective facts. Fourth, we did not perform a specific classification of thyroidectomy (total thyroidectomy, subtotal thyroidectomy, thyroid lobectomy) and failed to find an association between surgical procedures and postoperative hematoma. Fifth, the studies included in our meta-analysis did not describe whether hemostatic devices such as the Ligasure vessel sealing system and ultrasonic scalpels were used, and therefore, we could not judge whether the new type of hemostatic equipment(e.g. ultrasonic scapel) would reduce the incidence of hematoma. Finally, most of the included studies originated from European and American regions, which might lead to selective bias.

\section{Conclusions}

Our study identified several risk factors for neck hematoma requiring surgical re-intervention after thyroidectomy, including male gender, age, Graves disease, hypertension, antithrombotic agent use, thyroid procedures in low-volume hospitals, previous thyroid operation, bilateral thyroidectomy, and neck dissection. To minimize the occurrence of postoperative hematoma, extensive postoperative monitoring should be performed in patients with multiple identifiable risk factors, particularly when thyroidectomy is performed on an outpatient basis.

\section{Abbreviations}

Cl: Confidence interval; CNKI: China national knowledge infrastructure; COPD: Chronic obstructive pulmonary disease; MD: Mean difference; NOS: Newcastle Ottawa Scale; OR: Odds ratio; PRISMA: Preferred Reporting Items for Systematic Review and Meta-Analyses

\section{Acknowledgements}

We would like to thank the native English speaking scientists of Elixigen Company (Huntington Beach, California) for editing our manuscript. 


\section{Authors' contributions}

CF and XZ were major contributors to writing the manuscript, and they contributed equally to this work. CF and XZ were involved in the design of the study and drafting the manuscript. ML and $\mathrm{HL}$ collected and analysed data. GS, YZ and JS critically revised the manuscript till the final version was reached. All the authors read and approved the final manuscript.

\section{Funding}

This work was supported by the Health and Family Planning Commission of Fujian Province, China [grant number 2014-CXB-38] and the Technology Bureau of Xiamen City, China [grant number 3502Z20154005].

\section{Availability of data and materials}

All data generated or analyzed during this study are available from all the included studies from PubMed and CNKI databases.

\section{Ethics approval and consent to participate}

Not applicable.

\section{Consent for publication}

The manuscript is approved for publication by all the authors.

\section{Competing interests}

The authors declare that they have no competing interests.

\section{Author details}

'Department of Gastrointestinal Surgery III, Xiamen Cancer Hospital, First Affiliated Hospital of Xiamen University, 55 Zhenhai Road, Xiamen 361003, China. ${ }^{2}$ Department of Hepatobiliary Surgery, First Affiliated Hospital of Xiamen University, Xiamen, China. ${ }^{3}$ Department of Ultrasound, First Affiliated Hospital of Xiamen University, 55 Zhenhai Road, Xiamen 361003, China. ${ }^{4}$ Department of Hepatic Surgery and Liver transplantation Center, Third Affiliated Hospital of Sun Yat-sen University, Guangzhou, China.

Received: 7 January 2018 Accepted: 9 July 2019

Published online: 24 July 2019

\section{References}

1. Haugen BR. 2015 American Thyroid Association management guidelines for adult patients with thyroid nodules and differentiated thyroid cancer: what is new and what has changed? Thyroid Off J Am Thyroid Assoc. 2016;26(1):1.

2. Suzuki $\mathrm{S}$, et al. Factors associated with neck hematoma after thyroidectomy: a retrospective analysis using a Japanese inpatient database. Medicine (Baltimore). 2016:95(7):e2812.

3. Perera $\mathrm{M}$, et al. Risk factors for post-thyroidectomy haematoma. J Laryngol Otol. 2016:130(Suppl 1):S20-5.

4. Oltmann SC, et al. Antiplatelet and anticoagulant medications significantly increase the risk of postoperative hematoma: review of over 4500 thyroid and parathyroid procedures. Ann Surg Oncol. 2016;23(9):2874-82.

5. Narayanan S, et al. An evaluation of postoperative complications and cost after short-stay thyroid operations. Ann Surg Oncol. 2016;23(5):1440-5.

6. Liu J, et al. Risk factors for and occurrence of postoperative cervical hematoma after thyroid surgery: a single-institution study based on 5156 cases from the past 2 years. Head Neck. 2016;38(2):216-9.

7. Sorensen KR, Klug TE. Routine outpatient thyroid surgery cannot be recommended. Dan Med J. 2015;62(2):A5016.

8. Hardman JC, et al. Re-operative thyroid surgery: a 20-year prospective cohort study at a tertiary referral centre. Eur Arch Otorhinolaryngol. 2015;272(6):1503-8

9. Dehal A, et al. Risk factors for neck hematoma after thyroid or parathyroid surgery: ten-year analysis of the nationwide inpatient sample database. Perm J. 2015:19(1):22-8.

10. Weiss $A$, et al. Risk factors for hematoma after thyroidectomy: results from the nationwide inpatient sample. Surgery. 2014;156(2):399-404.

11. Dixon $J \mathrm{~L}$, et al. A novel method for the management of post-thyroidectomy or parathyroidectomy hematoma: a single-institution experience after over 4,000 central neck operations. World J Surg. 2014;38(6):1262-7.

12. Chen $\mathrm{E}_{\text {, et }}$ al. Risk factors target in patients with post-thyroidectomy bleeding. Int J Clin Exp Med. 2014;7(7):1837-44.
13. Vassiliou I, et al. Total thyroidectomy as the single surgical option for benign and malignant thyroid disease: a surgical challenge. Arch Med Sci. 2013;9(1):74-8.

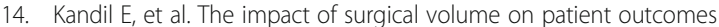
following thyroid surgery. Surgery. 2013;154(6):1346-52 discussion 1352-3.

15. Promberger $\mathrm{R}$, et al. Risk factors for postoperative bleeding after thyroid surgery. Br J Surg. 2012;99(3):373-9.

16. Lang $\mathrm{BH}$, Yih $\mathrm{PC}$, Lo CY. A review of risk factors and timing for postoperative hematoma after thyroidectomy: is outpatient thyroidectomy really safe? World J Surg. 2012;36(10):2497-502.

17. Calo $P G$, et al. Risk factors in reoperative thyroid surgery for recurrent goitre: our experience. G Chir. 2012;33(10):335-8.

18. Agarwal A, et al. Clinicopathological profile, airway management, and outcome in huge multinodular goiters: an institutional experience from an endemic goiter region. World J Surg. 2012;36(4):755-60.

19. Calo $P G$, et al. Postoperative hematomas after thyroid surgery. Incidence and risk factors in our experience. Ann Ital Chir. 2010:81(5):343-7.

20. Godballe C, et al. Post-thyroidectomy hemorrhage: a national study of patients treated at the Danish departments of ENT head and neck surgery. Eur Arch Otorhinolaryngol. 2009;266(12):1945-52.

21. Shih ML, et al. Thyroidectomy for Hashimoto's thyroiditis: complications and associated cancers. Thyroid. 2008;18(7):729-34.

22. Leyre $\mathrm{P}$, et al. Does the risk of compressive hematoma after thyroidectomy authorize 1-day surgery? Langenbeck's Arch Surg. 2008;393(5):733-7.

23. Bergenfelz $\mathrm{A}$, et al. Complications to thyroid surgery: results as reported in a database from a multicenter audit comprising 3,660 patients. Langenbeck's Arch Surg. 2008;393(5):667-73.

24. Lefevre $\mathrm{JH}$, et al. Reoperative surgery for thyroid disease. Langenbeck's Arch Surg. 2007:392(6):685-91.

25. Ozlem N, et al. Should the thyroid bed be drained after thyroidectomy? Langenbeck's Arch Surg. 2006;391(3):228-30

26. Gaujoux S, et al. Extensive thyroidectomy in Graves' disease. J Am Coll Surg. 2006;202(6):868-73.

27. Chiang FY, et al. Morbidity after total thyroidectomy for benign thyroid disease: comparison of Graves' disease and non-Graves' disease. Kaohsiung J Med Sci. 2006;22(11):554-9.

28. Sun GH, DeMonner S, Davis MM. Epidemiological and economic trends in inpatient and outpatient thyroidectomy in the United States, 1996-2006. Thyroid. 2013;23(6):727-33.

29. Terris DJ, et al. American Thyroid Association statement on outpatient thyroidectomy. Thyroid Off J Am Thyroid Assoc. 2013;23(10):1193-202.

30. Moher D, et al. Preferred reporting items for systematic reviews and meta-analyses: the PRISMA statement. Open Med. 2009;3(3):e123.

31. Wells GA, et al. The Newcastle-Ottawa scale (NOS) for assessing the quality of non-randomized studies in meta-analysis. Appl Eng Agric. 2014;18(6): p. págs):727-34

32. Stang A. Critical evaluation of the Newcastle-Ottawa scale for the assessment of the quality of nonrandomized studies in meta-analyses. Eur J Epidemiol. 2010;25(9):603-5.

33. Li Z, et al. Growth hormone replacement therapy reduces risk of cancer in adult with growth hormone deficiency: a meta-analysis. Oncotarget. 2016; 7(49):81862-9.

34. He $X$, et al. Thyroid antibodies and risk of preterm delivery: a meta-analysis of prospective cohort studies. Eur J Endocrinol. 2012;167(4):455-64.

35. Harding J, et al. Thyroid surgery: postoperative hematoma--prevention and treatment. Langenbeck's Arch Surg. 2006;391(3):169-73.

36. Hegedüs L. Clinical practice. The thyroid nodule. N Engl J Med. 2004; 351(17):1764.

37. Lin DZ, et al. Risk prediction and clinical model building for lymph node metastasis in papillary thyroid microcarcinoma. Oncotargets Ther. 2016:9:5307.

38. Bahn Chair RS, et al. Hyperthyroidism and other causes of thyrotoxicosis: management guidelines of the American Thyroid Association and American Association of Clinical Endocrinologists. Endocr Pract Off J Am Coll Endocrinol Am Assoc Clin Endocrinol. 2011;17(3):456

39. Singer PA, et al. Treatment guidelines for patients with hyperthyroidism and hypothyroidism. Standards of care committee, American Thyroid Association. JAMA. 1995:273(10):808-12.

40. Ross DS, et al. 2016 American Thyroid Association guidelines for diagnosis and Management of Hyperthyroidism and other causes of thyrotoxicosis. Thyroid Off J Am Thyroid Assoc. 2016;26(10):1343-421. 
41. Samona S, Hagglund K, Edhayan E. Case cohort study of risk factors for post-thyroidectomy hemorrhage. Am J Surg. 2016;211(3):537-40.

42. Tartaglia F, et al. Complications in total thyroidectomy: our experience and a number of considerations. Chir Ital. 2003;55(4):499.

43. Lee HS, et al. Patterns of post-thyroidectomy hemorrhage. Clin Exper Otorhinolaryngol. 2009;2(2):72.

44. Palestini N, et al. Post-thyroidectomy cervical hematoma. Minerva Chir. 2005; 60(1):37.

45. Robertebadi H, Le GG, Righini M. Use of anticoagulants in elderly patients: practical recommendations. Clin Interv Aging. 2009;4(1):165.

46. Sayaka S, et al. Factors associated with neck hematoma after thyroidectomy: a retrospective analysis using a Japanese inpatient database. Medicine. 2016;95(7):e2812

47. Callahan MA, et al. Influence of surgical subspecialty training on in-hospital mortality for gastrectomy and colectomy patients. Ann Surg. 2003;238(4):629.

48. Lien YC, Huang MT, Lin HC. Association between surgeon and hospital volume and in-hospital fatalities after lung cancer resections: the experience of an Asian country. Ann Thorac Surg. 2007;83(5):1837-43.

49. Sosa JA, et al. The importance of surgeon experience for clinical and economic outcomes from thyroidectomy. Ann Surg. 1998;228(3):320.

50. Shaha AR. Revision thyroid surgery - technical considerations. Otolaryngol Clin N Am. 2008:41(6):1169-83.

51. Shaha AR, Jaffe BM. Practical management of post-thyroidectomy hematoma. J Surg Oncol. 1994:57(4):235-8.

52. Kristoffersson A, Sandzén B, Järhult J. Drainage in uncomplicated thyroid and parathyroid surgery. Br J Surg. 1986;73(2):121-2.

53. Peix $J \mathrm{~L}$, et al. Drainage after thyroidectomy: a randomized clinical trial. Int Surg. 1992;77(2):122

54. Schoretsanitis $\mathrm{G}$, et al. Does draining the neck affect morbidity following thyroid surgery? Am Surg. 1998;64(8):778-80.

\section{Publisher's Note}

Springer Nature remains neutral with regard to jurisdictional claims in published maps and institutional affiliations.

Ready to submit your research? Choose BMC and benefit from:

- fast, convenient online submission

- thorough peer review by experienced researchers in your field

- rapid publication on acceptance

- support for research data, including large and complex data types

- gold Open Access which fosters wider collaboration and increased citations

- maximum visibility for your research: over $100 \mathrm{M}$ website views per year

At $\mathrm{BMC}$, research is always in progress.

Learn more biomedcentral.com/submissions 